\title{
FLUJOS DE EFECTIVO FUTUROS PARA EMPRESAS DEL SECTOR COMERCIAL DEL VALLE DEL CAUCA DURANTE EL PERIODO 1995 - $2014^{1}$
}

\author{
Gabriel Eduardo Escobar Arias ${ }^{2}$ \\ Jorge Iván Osorio Mejía ${ }^{3}$
}

Para citar este artículo: Escobar, G. y Osorio, J. (2016). Flujos de efectivo futuros para empresas del sector comercial del valle del cauca durante el periodo 1995 2014. Inquietud Empresarial. Vol. XVI (1), 55-79

Fecha de recepción: 9 de octubre de 2015

Fecha de aceptación: 18 de julio de 2016

\footnotetext{
${ }^{1}$ Este artículo es resultado de la investigación titulada "Crecimiento económico y flujos de efectivo en las empresas del sector comercial de Antioquia, Caldas, Cundinamarca, Santander y Valle". Las entidades que financiaron dicho proceso investigativo fueron la Universidad Nacional de Colombia sede Manizales y la Universidad Autónoma de Manizales. La investigación se realizó durante los años 2014 - 2015.

${ }^{2}$ Gabriel Eduardo Escobar Arias, docente Universidad Nacional de Colombia sede Manizales y Universidad Autónoma de Manizales, miembro del grupo de investigación en empresariado de la Universidad Autónoma de Manizales. Economista candidato a doctor en el programa de ingeniería - industria y organizaciones de la Universidad Nacional de Colombia. E-mail: gabrieledoescobar@gmail.com

${ }^{3}$ Jorge Iván Osorio Mejía, estudiante MBA (Cohorte VI) Universidad Autónoma de Manizales, Ingeniero Civil de la Universidad Nacional de Colombia sede Manizales. E- mail: jiom39@yahoo.com.mx
} 


\title{
Resumen
}

El principal objetivo fue determinar la relación entre el crecimiento económico y los flujos de efectivo de las empresas del sector industrial para el departamento del Valle del Cauca y luego con estos resultados poder realizar predicciones para los futuros flujos de efectivo. Dentro de la metodología, se obtuvieron los estados financieros de las empresas industriales del Valle del Cauca que reportan estados financieros a la Superintendencia de Sociedades durante el año 1995, las cuales ascendieron a 225, siendo este número de empresas la población inicial, sin embargo, el estudio solo se le pudo aplicar a 45 empresas que fueron aquellas que reportaron de forma continua sus estados financieros durante los años 1995 2014 a la Superintendencia de Sociedades de Colombia. A este número de empresas se le calcularon los flujos de efectivo de operación y financiación con los cuales se estableció la relación estadística con el crecimiento de la economía regional durante el mismo periodo de tiempo, datos que se obtuvieron del Departamento Nacional de Estadística (DANE) de Colombia. Los principales resultados obtenidos fueron la relación estadística encontrada entre los flujos de efectivo de operación y el crecimiento de la economía regional ascendió al 0,48, resultado bastante insatisfactorio inicialmente para poder realizar predicciones, pero al sumarle a los flujos de efectivo de operación, la financiación; la relación estadística aumentó a 0,77 , dato más preciso para poder realizar la predicción de los futuros flujos de efectivo. La principal conclusión fue que, al existir una relación directa y alta entre los flujos de efectivo de las empresas del sector industrial del Departamento del valle del Cauca y el crecimiento económico regional, se pueden realizar predicciones de los futuros flujos de efectivo para las empresas del sector, de tal forma que gremios y empresarios puedan realizar una mejor planeación financiera al interior de sus organizaciones.

Palabras clave: Flujos de efectivo, correlación, crecimiento económico y predicción.

\section{Future cash flows for companies of the commercial sector of Valle del Cauca during the period of $1995-2014$}

\begin{abstract}
The main objective of this study, was to determine the relationship between economic growth and cash flows of companies at the industrial sector of the department of Valle del Cauca. Then, with these results, to be able to make predictions for the future cash flows. Within the methodology, there were obtained the financial statements of the industrial companies of Valle del Cauca, which report financial statements to the Superintendency of Corporations during
\end{abstract}


1995 , which increased to 225 , being this number companies of the initial population. However, the study was applied to 45 companies that were those that continuously reported their financial statements during the years 1995 - 2014 to the Superintendence of Corporations of Colombia. To this number of companies, it was calculated the operating and financing cash flows with which the statistical relationship was established with the growth of the regional economy during the same period of time, data that were obtained from the National Department of Statistics (DANE) from Colombia. The main results obtained were the statistical relationship between operating cash flows and the growth of the regional economy that increased to 0.48 , a rather unsatisfactory result to make predictions at the beginning, but when added to the cash flows of operation, the financing; the statistical ratio increased to 0.77 , a more accurate data to be able to predict future cash flows. The main conclusion was that, since there is a direct and high relationship between the cash flows of companies at the industrial sector of the department of Valle del Cauca and regional economic growth, it is possible to make predictions of future cash flows for Sector. That is why guilds and entrepreneurs can realize a better financial planning within their organizations.

Key words: cash flows, correlation, economic growth and prediction.

\section{Fluxos de caixa futuros para fins comerciais EMPRESAS CAUCA VALLEY sector durante o período 1995 - 2014}

\section{Resumo}

O principal objetivo foi determinar a relação entre o crescimento económico e os fluxos de caixa das empresas do sector industrial para o departamento de Valle del Cauca e, em seguida, estes resultados para fazer previsões para os fluxos de caixa futuros. Dentro da metodologia, foram obtidos das demonstrações financeiras das empresas industriais de Valle del Cauca relatórios demonstrações financeiras para a Superintendência de Empresas, em 1995, que ascenderam a 225 , e este número de empresas a população inicial, no entanto, o estudo foi capaz de aplicar apenas 45 empresas foram as que continuamente relatou suas demonstrações financeiras para os anos de 1995 a 2014 à Superintendência de sociedades de Colômbia. Este número de empresas foi estimada fluxos de caixa operacionais e de financiamento com que a relação estatística com o crescimento da economia regional foi definido durante o mesmo período de tempo, os dados foram obtidos junto ao Departamento Nacional de Estatística (DANE) da Colômbia. Os principais resultados obtidos foram a relação estatística encontrada entre o fluxo de caixa operacional e crescimento da economia regional ascendeu a 0,48 , resultado bastante insatisfatório inicialmente para fazer previsões, mas quando adicionado a fluxos de caixa operacionais, a financiamento; relação 
estatística aumentou para 0,77 , para realizar uma previsão mais precisa dos futuros dados de fluxos de caixa. A principal conclusão foi que, como não há uma relação direta e alta entre os fluxos de caixa das empresas do setor industrial do departamento de Valle del Cauca e do crescimento económico regional, podemos fazer previsões de fluxos de caixa futuros para as empresas sector de modo a que os sindicatos e os empregadores podem fazer um melhor planejamento financeiro dentro de suas organizações.

Palavras chave: os fluxos de caixa, correlação e previsão de crescimento económico.

\section{Introducción}

En procesos investigativos anteriores se analizaron los flujos de efectivo de algunas empresas del departamento de Caldas, Risaralda y Quindío para el sector comercial, industrial, de servicios y agropecuario, entre 2002 y 2010 en sus estructuras de operación, financiación e inversión; Escobar (2014) y se determinó su relación con algunas variables macroeconómicas durante el mismo período. Los principales resultados se refieren a la demostración de la correlación positiva presentada entre las variables analizadas. De acuerdo con lo anterior, se indagó en profundidad sobre la relación que pueden presentar variables como el crecimiento económico y los flujos de efectivo y su incidencia en estos, de forma que se pueda realizar su predicción para empresas del sector comercial en el Valle del Cauca.

Algunos autores han presentado resultados sobre la predicción de los flujos de efectivo para períodos siguientes, a partir de variables como los flujos de efectivos históricos Catanach, Croll y Grinaker. (2000), Luo (2008) y Lorek (2014); los estados financieros, conformados por el balance general y el estado de resultados o situación económica Ou y Penman (1989), Stober (1992) y Lee (1996); el capital de trabajo Francis y Eason (2012); los ingresos o los costos Atwood et al. (2011), Sloan, (1996) y Stober (1993); y otros informes financieros redactados bajo normas internacionales de información financiera (NIIF), Lee (2011) y Legoria y Sellers (2005). Sin embargo, ninguno de ellos, en la búsqueda realizada, trabajó la predicción a través de la relación que pueda haber entre el crecimiento de la economía y los flujos de efectivo históricos. En este sentido se observa un vacío teórico entre la relación que puedan tener estas dos variables y la manera de conjugarlas para establecer una predicción de los flujos de efectivo a partir de estos resultados.

Los objetivos del trabajo fueron, en primer lugar, establecer la relación teórica y práctica entre las dos variables, y, en segundo lugar, establecer la correlación entre el crecimiento de la economía y los flujos de efectivo durante los años 1995-2014, para las empresas del sector comercial de la región señalada. Y, 
finalmente, plantear un sistema que permita proyectar los flujos de efectivo a partir de la variación que presenta el crecimiento de la economía, es decir, medir el porcentaje de variación que presentarán los flujos de efectivo ante la variación del PIB de la economía en la cual se basa el estudio. Esta observación se fundamenta en que la variable independiente será el crecimiento económico y la variable dependiente serán los flujos de efectivo.

Con los resultados obtenidos, los empresarios podrán tener información adicional para realizar sus pronósticos respecto a los flujos de efectivo, pues cuentan con información interna y externa y de esta forma podrán realizar una planeación financiera más adecuada para prever su capital de trabajo, las inversiones futuras, el pago de dividendos y de obligaciones financieras, entre otras actividades relacionadas con el desembolso o la adquisición de recursos monetarios o liquidez.

La investigación realizada fue de tipo cuantitativo en su parte inicial, pues se realizó una extracción de cifras como son los estados de los flujos de efectivo de las empresas que reportaron de forma continua esta información a la Superintendencia de Sociedades Supersociedades (2015) de Colombia durante los años 1995 - 2014, para la región mencionada. La información se recopiló y se presentó el acumulado de datos para el Valle del Cauca. Los datos del Producto Interno Bruto (PIB) se consultaron en la base de datos del Departamento Administrativo Nacional de Estadística -DANE, además de considerar las proyecciones del Banco Mundial, y así obtener el crecimiento económico de la región en el período mencionado. Con esta información, se estableció las correlaciones que presentaron las variables estudiadas, y se desarrolló el análisis de regresión estadística que permite establecer la predicción de los flujos de efectivo futuros para los años 2015, 2016 y 2017.

Se presentó, además, un análisis en el cual se estableció la relación que pueden tener algunos hechos económicos con los flujos de efectivo para el departamento del Valle del Cauca y durante el mismo tiempo, de tal forma que se pueda demostrar que la correlación entre las dos variables es posible explicarla de forma cuantitativa y cualitativa.

Uno de los principales problemas encontrados en el proceso investigativo fue la muestra a la cual se le aplica el modelo estadístico de regresión para predecir los futuros flujos de efectivo, puesto que para la región analizada se encontró un total de 225 empresas que pertenecen al sector comercial, pero de estas solamente 45 reportaron los estados de flujos de efectivo de forma continua ante la Superintendencia de Sociedades, esto se debió a que muchas de ellas desaparecieron, otras se fusionaron y otras no presentaron el reporte. 
Es necesario que el reporte sea continuo porque los datos en cero hacen que los datos estadísticos pierdan homogeneidad y tanto las correlaciones como las predicciones pierden validez.

\section{Metodología}

La información que se presenta en este artículo es de tipo cuantitativo, puesto que se realiza un análisis de cifras como los estados de flujos de efectivo de las empresas del departamento objeto del presente estudio, que pertenecen al sector comercial y que han reportado estados financieros a las Superintendencia de Sociedades entre 1995 y 2014.

La investigación es de carácter correlacional, por lo cual uno de sus objetivos fue establecer la relación que hay entre el crecimiento económico regional y los flujos de efectivo para las empresas del sector comercial en el Valle del Cauca. Se pretendió establecer en esta correlación el efecto de la variable independiente, que es el crecimiento económico, en la variable dependiente que son los flujos de efectivo.

Además, el trabajo es de carácter longitudinal, puesto que se evalúan varios periodos continuos en cada una de las variables que son el crecimiento económico y los flujos de efectivo. El periodo analizado es entre 1995 y 2014. Este trabajo, también tiene un carácter exploratorio puesto que se trata de establecer los futuros flujos de efectivo de las empresas estudiadas a través de la relación entre las variables crecimiento económico y flujos de efectivo.

Para la recolección de la información se utilizó la base de datos (SIREM) que es operada y suministrada de forma gratuita por la superintendencia de sociedades. Esta base de datos se encuentra en la página Web de la entidad (www.supersociedades.gov.co).

Para determinar el Producto Interno Bruto proyectado, se tomó la información suministrada por el Banco Mundial, entidad que tiene proyecciones de crecimiento PIB estimadas hasta el año 2017.

Para determinar la población se estableció el número de empresas del sector comercial del Valle del Cauca, que reportaron estados financieros a la Superintendencia de Sociedades durante 1995, y se obtuvieron 225 empresas.

Para determinar la muestra, se tomaron las empresas que reportaron estados financieros a la Superintendencia de Sociedades entre 1995 y 2014 de forma continua. Se decidió de esta manera porque en los periodos en los cuales no hay reporte, para la elaboración del aplicativo estadístico se tendría un dato cero, que afectaría los resultados de la correlación estadística. 
Para el Valle del Cauca, objeto del presente estudio, se obtuvo el siguiente número de empresas:

Tabla 1. Empresas del sector comercial que reportaron estados financieros para el Valle del Cauca, de forma continua a la Superintendencia de Sociedades durante el periodo $1995-2014$.

\begin{tabular}{|c|c|}
\hline Departamento / región & Número de empresas \\
\hline Valle del Cauca & 45 \\
\hline
\end{tabular}

Fuente: Elaboración propia con datos obtenidos de la Superintendencia de Sociedades.

Operatividad de la investigación:

Para el logro de los objetivos, se operó de la siguiente manera:

- Se contó con los históricos del PIB desde 1995 hasta 2014.

- Se calcularon los flujos de efectivo de las empresas estudiadas a partir de los estados financieros Balance General y Estado de Resultados, datos suministrados por la Superintendencia de Sociedades entra 1995 y 2014.

- Se obtuvo la sumatoria de los flujos de efectivo de las 45 empresas del sector comercial de Valle del Cauca, a las cuales se les aplicó el modelo estadístico.

- A través del software estadístico SPSS se estableció el grado de asociación lineal y no lineal entre el PIB y los flujos de efectivo (relación), dato que entregó información importante para determinar el modelo econométrico que se utilizó para establecer los flujos de efectivo futuros.

- Se realizó una descripción de los hechos económicos ocurridos para cada región entre 1995 y 2014 con el objeto de determinar la relación de estos con los resultados de los flujos de efectivo.

- Para la estimación de los flujos de efectivo futuros para el período, de acuerdo con la estimación del PIB proyectado, se realizó mediante un modelo de regresión lineal de los flujos de efectivo en función del PIB de la siguiente forma:

F.E.E. $=$ Constante $+($ C x C.E. $)+$ Error

De donde:

F.E.E. = Flujo de efectivo esperado.

$\mathrm{C}=$ Coeficiente del PIB.

C.E. = Crecimiento económico esperado. 
Para la comprobación y la validez de la regresión de los datos y del modelo planteado, se realizaron pruebas estadísticas como La T de Student, que consiste en comprobar si "dos grupos difieren entre sí de manera significativa respecto a sus medias" Sampieri et al. (2006). Y Para determinar la validez del modelo, el nivel de significancia de la prueba debe ser menor a 0,05 Creswell (2005).

Otra prueba que se debe aplicar al modelo de regresión es la de Fisher, que examina la igualdad entre las varianzas poblacionales de dos variables con distribución normal. Para la aceptación de la prueba bajo este parámetro, su nivel de significancia debe ser menor a 0,05 Fisher (1925). La tercera prueba es el coeficiente de determinación, que muestra el valor que puede explicar el efecto de la variable independiente en la variable dependiente. En la medida que esta se acerque más a 1 , significa que hay mayor grado de correlación y por eso los datos son más homogéneos para realizar modelos de predicción Creswell (2005).

Por su parte, Sampieri et al. (2006) utiliza una escala para determinar el grado de correlación, indicando que, cuando el coeficiente de correlación está entre 0,25 y 0,50 es una correlación media, cuando está entre 0,50 y 0,75 es una correlación positiva, y mayor a estos intervalos se considera que la correlación es muy alta.

La siguiente prueba estadística para determinar el modelo de regresión lineal es la normalidad, que muestra si los datos de las variables analizadas tienen una distribución normal, en la medida que su nivel de significancia sea menor a 0,05 indica que los datos son homogéneos, es decir, que están dentro de la distribución normal. Por lo tanto, los datos son confiables para construir modelos de predicción León y Montero (2003).

La última prueba que se utiliza para comprobar la validez del modelo es la Homocedasticidad, que consiste en determinar la igualdad que presentan las varianzas de las variables analizadas. Para determinar que la prueba es válida, su resultado debe ser superior al 5\% de acuerdo con la prueba de Levene. Aranaz (2002).

Inicialmente se utilizó el modelo de regresión lineal porque determina el efecto de una variable sobre otra Sampieri et al. (2006), de modo que, permite determinar el efecto del crecimiento económico, como variable independiente, en los flujos de efectivo como variable dependiente, sin embargo, los resultados no permitieron la validación estadística del modelo presentado. Dado que el software estadístico utilizado permite obtener resultados de correlación de diferentes modelos de regresión, se procedió a la búsqueda del modelo que mejor representara el comportamiento de las variables, para el caso de este estudio el modelo que presentó un mejor ajuste en los coeficientes de correlación y de determinación correspondió a la regresión cúbica, que se clasifica dentro del modelo polinómico (en este cado de tercer orden) y pertenece a un tipo del 
modelo no lineales (no obstante, este modelo polinómico que procede del modelo lineal). La relación observada entre las variables se distribuye alrededor de una curva y la ecuación de dicha curva se determina a partir del modelo no lineal seleccionado Sancho y Serrano (2006). Acto seguido se validó estadísticamente el modelo con la variable dependiente ya transformada y analizando la normalidad que presentan los residuos arrojados por el modelo.

\section{Resultados}

La inversión en capital es la base para el crecimiento económico de un país Solow (1956), y a partir de este elemento se da la relación entre los flujos de efectivo y el crecimiento económico, pues hay un conector entre estas dos variables que es la liquidez como se muestra a continuación:

El flujo de efectivo, es decir, la liquidez, indica la disponibilidad de recursos con los que cuenta una empresa para atender sus obligaciones financieras, realizar inversiones o distribuir utilidades García (1999). Por lo tanto, la inversión hace parte del flujo de efectivo del cual se generan los recursos para el desarrollo de la actividad de las empresas.

Las inversiones privadas y públicas requieren grandes desembolsos de dinero y en muchas ocasiones ni los empresarios ni los gobiernos disponen de fondos para efectuarlos. Por este motivo, el sistema financiero hace parte de dicha relación y se encarga de canalizar los recursos suficientes para que los fondos sean trasladados a la inversión.

El flujo de efectivo determina la liquidez que se puede llevar a la inversión pues, en muchos casos, el flujo no contiene los recursos necesarios para su realización, y por eso los empresarios recurren al sistema financiero para conseguir la liquidez necesaria para sus inversiones Levine (1997). Pero para que esto se pueda dar, las empresas deben tener la suficiente liquidez para retornar estos dineros prestados a las entidades financieras a unas tasas de interés adecuadas en un tiempo prudente. 
Gráfica 1: Movilidad de la inversión.

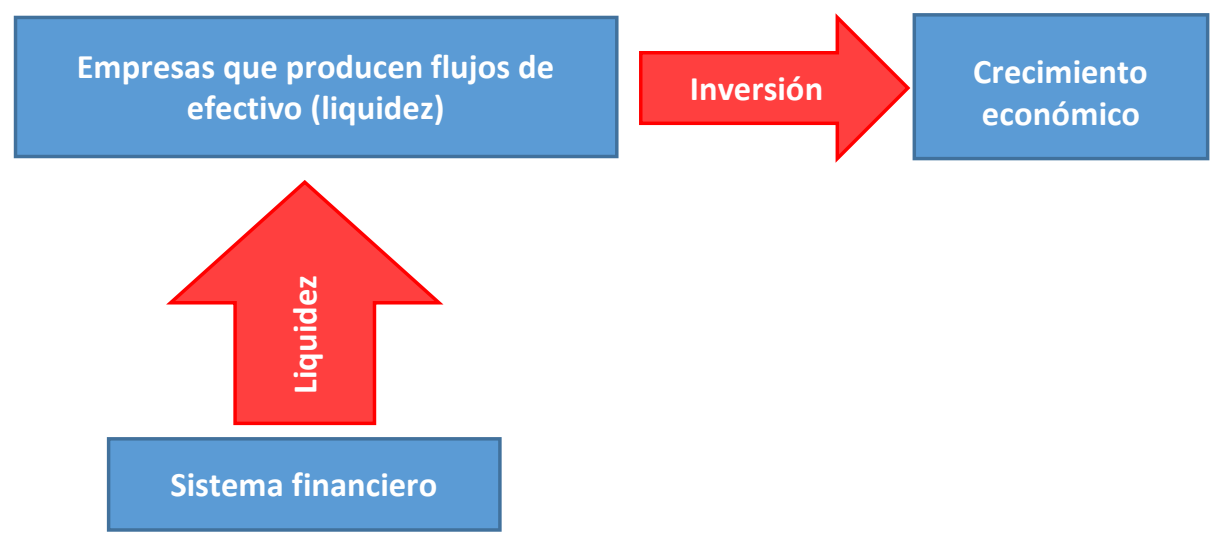

Fuente: Elaboración propia.

El sistema financiero inyecta liquidez a las empresas, y estos recursos son invertidos en acumulación de capital y en innovación tecnológica que aportan al crecimiento de la economía. El flujo de efectivo es liquidez que se puede utilizar para invertir en acumulación de capital y en innovación tecnológica, que suelen demandar grandes cantidades de recursos monetarios. Por este motivo, que los empresarios se apoyan en el sistema financiero para hacer estas inversiones. Por esto, las entidades financieras hacen parte del crecimiento económico de un país Levine (1997).

Algunos autores sostienen que la movilidad de estos recursos por el sistema financiero, al ser trasladados a las empresas para que estas realicen sus inversiones, se promueve el crecimiento económico de los países.

Hicks (1969) demostró que el sistema financiero fue la base fundamental para el comienzo de la industrialización en Inglaterra, pues canalizó los recursos para la inversión de grandes obras en este país. Los productos y las invenciones ya estaban elaboradas y operaban desde años anteriores y el crecimiento económico aún no había llegado. Sin embargo, cuando el sistema financiero inyectó la liquidez necesaria a través del mercado de capitales, los instrumentos financieros fueron los impulsadores de las inversiones de largo plazo que la economía necesitaba.

De igual manera, Shumpeter (1912) sostiene que el sistema financiero y en particular los bancos son entidades que promueven la inversión tecnológica. El sistema identifica los empresarios que están mejor preparados para llevar a cabo innovaciones y crear nuevos productos, y les otorga los recursos necesarios para 
que hagan realidad sus políticas empresariales. Para él, los bancos son entidades cuya tarea es identificar empresarios que creen mecanismos de producción eficaces y es a estos quienes con mayor facilidad se les otorgarán los recursos monetarios (liquidez), puesto que suelen obtener mejores resultados financieros que otros empresarios que no están preparados. En esta forma, los riesgos de prestar recursos disminuyen porque la probabilidad de recuperar los dineros invertidos en este tipo de empresas es mayor que las que no tienen la capacidad de innovar y mejorar los procesos productivos. Dice también el autor que, en la medida que las empresas cuenten con los recursos necesarios para realizar sus inversiones en innovación, tecnología y nuevos productos, estas nuevas capacidades impulsan el desarrollo económico de un país.

Robinson (1952), como los dos autores anteriores, sostiene que, a medida que se va dando el crecimiento económico de un país, se van creando las condiciones necesarias para que el sistema financiero provea los productos y servicios financieros adecuados para las nuevas tendencias que se producen en la economía. Con los años, el sistema financiero ha sido el encargado de ofrecer la liquidez necesaria para que los empresarios con nuevos productos y servicios financieros puedan hacer realidad sus inversiones y puedan inyectar capital en tecnología para mejorar sus procesos productivos y ser más competitivos.

Por su parte, North (1990) señala cómo el sistema bancario es uno de los autores principales para el logro del desarrollo y el crecimiento de un país, en la medida que las condiciones financieras de la economía sean mejores o más apropiadas se tendrá un mayor crecimiento, puesto que las entidades bancarias realizarán sus desembolsos de recursos más fácilmente y en mayores cantidades porque, al haber un sistema financiero más eficiente y consolidado, las entidades financieras tendrán mayor información sobre donde colocar sus recursos con menor riesgo y con más altas posibilidades de recuperación de su cartera. La información que tengan las entidades financieras sobre sus clientes y sobre los proyectos en los que pueden invertir, tendrán mejores condiciones financieras, lo que se traduce en un mayor crecimiento económico, en la medida que más cantidad de recursos se puedan canalizar hacía la inversión por las empresas que hacen uso de estos recursos monetarios.

Además, Atje y Jovanovic (1993) demuestran que, en la medida que se dé un buen desarrollo del sistema financiero en una economía, se da un mayor crecimiento económico, puesto que, cuando los empresarios usan los recursos que el sistema financiero les facilita producen necesariamente a una mayor inversión. Las inversiones en el mercado de capitales se incrementan al tener mayor liquidez, y al haber un sistema financiero más desarrollado los riesgos de inversión disminuyen incentivando el incremento de las inversiones y un mayor crecimiento económico. 
Bencivenga et al. (1995) demostraron que la inyección de una mayor liquidez por el sistema financiero a las empresas para la inversión produce crecimiento de la economía. Los autores señalan que los costos de transacción afectan de forma directa la inyección de liquidez que se destina a la inversión. De esta manera, en la medida que los costos de transacción financieros son más altos, se genera un menor incentivo por los inversionistas a adquirir activos financieros o productos que emitan las entidades financieras. Y, al contrario, en la medida que los costos transaccionales disminuyan, los incentivos a invertir son mayores, y se inyecta mayor liquidez al sistema, lo que produce mayor inversión y por tanto mayor crecimiento económico.

Para el análisis cualitativo se relacionan los principales hechos económicos ocurridos durante el periodo 1995 - 2014 para el Valle del Cauca, obteniendo los siguientes resultados:

A finales de los años noventa se calificó el desempeño departamental en cuanto al crecimiento se refiere como una década perdida ya que el crecimiento promedio apenas alcanzó un 0,77\% DANE (2014). Siendo las ramas de actividad más afectadas la industria y el sector agrícola. Mientras que otros sectores mostraron mejor dinamismo como lo fueron los servicios y la construcción.

Durante los primeros años de la década de los dos mil, el sector agrícola e industrial mostraron unos resultados más alentadores gracias al incremento en las exportaciones. Otro sector que jalonó los buenos resultados fue el de servicios financieros, quienes apoyaron a través del crédito este impulso en las exportaciones generando así mismo un incremento en las utilidades de los bancos.

A pesar de la crisis ocurrida en Venezuela, los empresarios reaccionaron de la región de forma acertada exportando sus productos a otros países, gracias a lo oportunidades que se generaron por la ley de preferencias arancelarias andinas y de erradicación de cultivos ilícitos (APTDEA).

Al final de la década los ingresos de las empresas comenzaron a decaer la igual que le crecimiento de la economía, debido a la crisis financiera internacional la cual tuvo repercusiones a nivel mundial y tanto Colombia como el departamento del Valle del cauca no fueron ajenos a estos efectos que se presentaron en la economía mundial.

A partir de la segunda década de los años dos mil, nuevamente la economía regional comenzó a presentar mejores resultados gracias a que se generaron nuevos negocios de exportación con aquellos países que se tenían relaciones comerciales desde años atrás.

Los eventos anteriormente nombrados coinciden con los resultados de los flujos de efectivo, es decir, en aquellos periodos donde se presentaba un buen 
FLUJOS DE EFECTIVO FUTUROS PARA EMPRESAS DEL SECTOR COMERCIAL DEL VALLE DEL CAUCA DURANTE EL PERIODO 1995 - 2014

desempeño de la economía de igual forma se comportaron los flujos de efectivo, demostrando de esta forma la relación existente entre las variables estudiadas.

Para establecer la correlación cuantitativa entre las dos variables se trabajó con los siguientes datos:

Tabla 2. Datos para el análisis estadístico.

\begin{tabular}{|c|c|c|c|c|}
\hline Período & $\begin{array}{l}\text { a. Índice } \\
\text { Deflactor }\end{array}$ & $\begin{array}{l}\text { c. Valor del } \\
\text { PIB } \\
\text { deflactado a } \\
\text { precios base } \\
\text { año } 2008\end{array}$ & $\begin{array}{l}\text { d. Crecimiento } \\
\text { económico para el } \\
\text { Valle del Cauca } \\
\text { durante los } \\
\text { períodos } 1996 \text { - } \\
2014\end{array}$ & $\begin{array}{l}\text { e. Flujos de efectivo } \\
\text { deflactados para el } \\
\text { valle del Cauca } \\
\text { durante el período } \\
1995 \text { - } 2014\end{array}$ \\
\hline 1995 & 31,23709 & 32.778 .930 & $-1,43 \%$ & (78.116.780) \\
\hline 1996 & 37,99651 & 32.312 .041 & $0,35 \%$ & (59.051.328) \\
\hline 1997 & 44,71589 & 32.426 .701 & $-0,41 \%$ & (205.665.329) \\
\hline 1998 & 52,18481 & 32.294 .982 & $-1,61 \%$ & $(66.744 .472)$ \\
\hline 1999 & 57,00236 & 31.778 .960 & $14,62 \%$ & 73.398 .837 \\
\hline 2000 & 61,98903 & 36.782 .315 & $0,62 \%$ & 67.802 .081 \\
\hline 2001 & 66,72893 & 37.009 .435 & $1,45 \%$ & 66.298 .519 \\
\hline 2002 & 71,39513 & 37.551 .581 & $1,94 \%$ & 33.384 .711 \\
\hline 2003 & 76,02913 & 38.285 .326 & $5,26 \%$ & 16.731 .169 \\
\hline 2004 & 80,20885 & 40.352 .156 & $2,05 \%$ & 102.459 .825 \\
\hline 2005 & 84,10291 & 41.188 .825 & $9,10 \%$ & 25.692 .526 \\
\hline 2006 & 87,86896 & 45.111 .493 & $6,98 \%$ & 24.671 .998 \\
\hline 2007 & 92,87228 & 48.375 .037 & $-0,57 \%$ & (44.022.739) \\
\hline 2008 & 100,00000 & 48.100 .000 & $4,50 \%$ & 131.784 .524 \\
\hline 2009 & 102,00181 & 50.313 .813 & $1,23 \%$ & 176.473 .992 \\
\hline 2010 & 105,23651 & 50.936 .695 & $3,77 \%$ & 74.073 .746 \\
\hline 2011 & 109,15740 & 52.893 .345 & $3,86 \%$ & 67.045 .442 \\
\hline 2012 & 111,81576 & 54.977 .044 & $4,67 \%$ & 93.768 .131 \\
\hline 2013 & 113,98254 & 57.604 .437 & $3,70 \%$ & 52.202 .794 \\
\hline 2014 & 114.38750 & 59.773 .176 & $-1,43 \%$ & $(78.116 .780)$ \\
\hline
\end{tabular}

Fuente: Elaboración propia con datos extraídos de la Superintendencia de Sociedades de Colombia y el Departamento Nacional de Estadística (DANE). 
Con los datos anteriores se procedió a realizar la regresión estadística obteniendo los siguientes resultados:

Tabla 3. Correlación estadística y coeficientes de determinación entre Producto Interno Bruto y flujos de efectivo de operación y financiación para el Valle del Cauca durante el período 1996 - 2014.

\begin{tabular}{|l|c|c|c|}
\hline $\begin{array}{c}\text { Departamento / } \\
\text { Ciudad }\end{array}$ & Modelo & $\begin{array}{c}\text { Coeficiente de } \\
\text { correlación (r) }\end{array}$ & $\begin{array}{c}\text { Coeficiente de } \\
\text { determinación }\left(\mathbf{r}^{2}\right)\end{array}$ \\
\hline \multirow{2}{*}{ Valle del Cauca } & $\begin{array}{c}\text { Regresión } \\
\text { lineal }\end{array}$ & 0,481 & 0,231 \\
\cline { 2 - 4 } & $\begin{array}{c}\text { Regresión } \\
\text { cúbica }\end{array}$ & 0,778 & 0,606 \\
\hline
\end{tabular}

Fuente: Elaboración propia con datos extraídos de la tabla número 2.

De acuerdo con las proyecciones de crecimiento del Banco Mundial para el crecimiento de la economía, se obtuvieron los siguientes datos:

Tabla 4. Proyecciones de crecimiento económico para Colombia para los años $2014-2017$.

\begin{tabular}{|c|c|}
\hline Período & $\begin{array}{c}\text { Crecimiento económico } \\
\text { proyectado }\end{array}$ \\
\hline 2014 & $4,6 \%$ \\
\hline 2015 & $3,5 \%$ \\
\hline 2016 & $3,9 \%$ \\
\hline 2017 & $4,2 \%$ \\
\hline
\end{tabular}

Fuente: Elaboración propia con datos extraídos del Banco Mundial (2015).

Con la información anterior se procedió a aplicar el modelo de regresión lineal, en el cual se obtuvo la siguiente información: para este proceso, la población fueron 45 empresas con las variaciones del PIB entre 1996 y 2014 y los respectivos flujos de efectivo para el mismo período, con los siguientes resultados. 
FLUJOS DE EFECTIVO FUTUROS PARA EMPRESAS DEL SECTOR COMERCIAL DEL VALLE DEL CAUCA DURANTE EL PERIODO 1995 - 2014

Tabla 5. Análisis estadístico descriptivo para el departamento del Valle del Cauca utilizando las variables crecimiento económico y flujos de efectivo.

\begin{tabular}{|c|c|c|}
\hline & $\begin{array}{c}\text { Crecimiento } \\
\text { económico (PIB) }\end{array}$ & $\begin{array}{c}\text { Flujos de efectivo de } \\
\text { operación y financiación }\end{array}$ \\
\hline $\begin{array}{c}\text { Número de } \\
\text { observaciones }\end{array}$ & 19 & 19 \\
\hline Media & $3,16 \%$ & 56.876 .072 \\
\hline Desviación estándar & $3,98 \%$ & $1,331 \mathrm{E}+8$ \\
\hline Mínimos & $-1,61 \%$ & -294.983 .343 \\
\hline Máximos & $14,62 \%$ & 205.284 .384 \\
\hline
\end{tabular}

Fuente: Elaboración propia.

De acuerdo con los datos de la tabla anterior, se puede determinar que El PIB entre 1995 y 2014 presenta un crecimiento medio de 3,16\% con una dispersión en los datos de $3,98 \%$, lo que comprueba que en este período hubo una alta variabilidad en el crecimiento económico, que se refleja en una disminución económica de $-1,61 \%$ hasta un crecimiento económico de 14,62\%, entre 1996 y 2014.

En todo caso, se establece para la media, con un intervalo de confianza del 95\% que va desde $1,24 \%$ hasta $5,08 \%$, valores útiles para trabajar en la predicción del flujo de efectivo entre 2015 y 2017. Al mismo tiempo, la variable flujos de efectivo, presentó una media de $\$ 56$ millones, con una variabilidad de $\$ 133$ millones, lo que indica que hay una dispersión alta entre los flujos de efectivo generados cada año. Esta situación se puede evidenciar con el amplio rango en que se encuentran los datos, que van desde $-\$ 294$ millones hasta \$205 millones en el mismo período. 
Para análisis de regresión el proceso utilizado fueron las mismas variables para establecer el modelo adecuado para la predicción de los flujos de efectivo y se obtuvieron los siguientes datos:

Tabla 6. Análisis de regresión estadístico para el departamento del Valle del Cauca entre los años 1996 - 2014.

\begin{tabular}{|l|r|}
\hline \multicolumn{1}{|c|}{ Variables estadísticas } & \multicolumn{1}{c|}{ Resultados } \\
\hline Constante & -13.639 .479 \\
\hline Coeficiente del PIB ${ }^{3}$ & 586.801 \\
\hline Coeficiente del PIB ${ }^{2}$ & -13.719 .261 \\
\hline Coeficiente del PIB & 84.552 .075 \\
\hline
\end{tabular}

Fuente: Elaboración propia.

De acuerdo con el resultado de la tabla anterior para la predicción de los flujos de efectivo futuros se establece la siguiente fórmula:

F.E.E. $=-13.639 .479+586.801 \times \mathrm{PIB}^{3}-13.719 .261 \times \mathrm{PIB}^{2}+84.552 .075 \times \mathrm{PIB}$

La constante significa que si el valor del PIB de un período es de cero $(0 \%)$, es decir, si el crecimiento es nulo, los flujos de efectivo de las empresas del sector comercial en conjunto tendrán un valor de $-\$ 13.639 .479$, los coeficientes del PIB significan que ante una variación del PIB de manera positiva o negativa, los flujos de efectivo aumentarán o disminuirán conforme el comportamiento de la función cúbica. 
FLUJOS DE EFECTIVO FUTUROS PARA EMPRESAS DEL SECTOR COMERCIAL DEL VALLE DEL CAUCA DURANTE EL PERIODO 1995 - 2014

Para determinar que el modelo presentado es válido estadísticamente para el departamento del Valle del Cauca, se realizaron las pruebas al modelo descrito en la metodología, obteniendo los siguientes datos:

Tabla 7. Pruebas estadísticas del modelo de regresión lineal para el departamento del Valle del Cauca.

\begin{tabular}{|c|c|c|c|c|}
\hline Prueba & $\begin{array}{c}\text { Resultado } \\
\text { de la } \\
\text { prueba }\end{array}$ & $\begin{array}{c}\text { Límites de la } \\
\text { prueba }\end{array}$ & $\begin{array}{c}\text { Aceptación } \\
\text { de la } \\
\text { prueba }\end{array}$ & $\begin{array}{c}\text { Rechazo } \\
\text { de la } \\
\text { prueba }\end{array}$ \\
\hline $\begin{array}{l}\text { T de Student } \\
\text { Significancia de la } \\
\text { prueba } \\
\text { Coeficiente de la } \\
\text { variable PIB }\end{array}$ & $\begin{array}{l}2,261 \\
3,7 \% \\
0,481\end{array}$ & $\begin{array}{c}\text { Significancia } \\
<5 \%\end{array}$ & $\mathrm{X}$ & \\
\hline $\begin{array}{l}\text { Fisher } \\
\text { Significancia de la } \\
\text { prueba }\end{array}$ & $\begin{array}{l}5,113 \\
3,7 \%\end{array}$ & $\begin{array}{c}\text { Significancia } \\
<5 \%\end{array}$ & $\mathrm{X}$ & \\
\hline $\begin{array}{l}\text { Coeficiente de } \\
\text { determinación }\end{array}$ & $23,1 \%$ & $\begin{array}{c}\text { Cerca al } \\
50 \%\end{array}$ & & $X$ \\
\hline $\begin{array}{l}\text { Normalidad } \\
\text { Significancia de la } \\
\text { prueba }\end{array}$ & $\begin{array}{l}1,335 \\
5,7 \%\end{array}$ & $>5 \%$ & $\mathrm{X}$ & \\
\hline Homocedasticidad & N.A. & $>5 \%$ & & $\mathrm{X}$ \\
\hline
\end{tabular}

Fuente: Elaboración propia.

De acuerdo con los resultados de la tabla anterior, el modelo no es válido para realizar la predicción de los flujos de efectivos futuros ante una variación determinada del PIB, puesto que no cumple todas las pruebas estadísticas planteadas. 
Por lo anterior se aplicaron diferentes modelos de regresión resultando que la mejor relación entre las variables analizadas corresponde a la regresión cúbica. Se obtuvieron los siguientes datos:

Tabla 8. Pruebas estadísticas del modelo de regresión cúbica para el departamento del Valle del Cauca.

\begin{tabular}{|c|c|c|c|c|}
\hline Prueba & $\begin{array}{c}\text { Resultado } \\
\text { de la } \\
\text { prueba }\end{array}$ & $\begin{array}{l}\text { Límites de } \\
\text { la prueba }\end{array}$ & $\begin{array}{l}\text { Aceptación } \\
\text { de la } \\
\text { prueba }\end{array}$ & $\begin{array}{c}\text { Rechazo } \\
\text { de la } \\
\text { prueba }\end{array}$ \\
\hline $\begin{array}{l}\text { T de Student } \\
\text { Significancia de la } \\
\text { prueba } \\
\text { Coeficiente de la } \\
\text { variable PIB }\end{array}$ & $\begin{array}{l}4,260 \\
0,1 \% \\
2,528\end{array}$ & $\begin{array}{c}\text { Significancia } \\
<5 \%\end{array}$ & $\mathrm{X}$ & \\
\hline $\begin{array}{l}\text { Fisher } \\
\text { Significancia de la } \\
\text { prueba }\end{array}$ & $\begin{array}{l}7,767 \\
0,2 \%\end{array}$ & $\begin{array}{c}\text { Significancia } \\
<5 \%\end{array}$ & $X$ & \\
\hline $\begin{array}{ll}\text { Coeficiente } & \text { de } \\
\text { determinación } & \end{array}$ & $52,7 \%$ & $\begin{array}{c}\text { Cerca al } \\
50 \%\end{array}$ & $X$ & \\
\hline $\begin{array}{l}\text { Normalidad } \\
\text { (Residuos) } \\
\text { Significancia de la } \\
\text { prueba }\end{array}$ & $\begin{array}{c}0,892 \\
35,2 \%\end{array}$ & $>5 \%$ & $X$ & \\
\hline Homocedasticidad & $8 \%$ & $>5 \%$ & $\mathrm{X}$ & \\
\hline
\end{tabular}

Fuente: Elaboración propia.

De acuerdo con los resultados de la tabla anterior, el modelo es válido para realizar la predicción de los flujos de efectivos futuros ante una variación determinada del PIB, puesto que cumple todas las pruebas estadísticas planteadas. 
En la aplicación al modelo y para determinar el efecto de la variación económica en los flujos de efectivo, se emplea la fórmula del modelo establecido y se prueba con diferentes variaciones en el PIB, para medir el cambio que presentan los flujos de efectivo. Para ello, se ensambló la formula y se realizó una simulación donde el PIB variaba en un punto porcentual y se obtuvieron los siguientes resultados:

Tabla 9. Resultados de la variación de los flujos de efectivo para el departamento del Valle del Cauca ante cambios en el PIB en un punto porcentual.

\begin{tabular}{|c|c|c|c|c|c|c|c|c|}
\hline Constante & $\begin{array}{c}\text { Coeficiente } \\
\text { PIB }^{3}\end{array}$ & $\begin{array}{c}\text { Coeficiente } \\
\text { PIB }^{2}\end{array}$ & $\begin{array}{c}\text { Coeficiente } \\
\text { PIB }\end{array}$ & $\begin{array}{c}\text { PIB } \\
\text { inicial }\end{array}$ & $\begin{array}{l}\text { PIB } \\
\text { final }\end{array}$ & $\begin{array}{c}\text { Valor del } \\
\text { flujo de } \\
\text { efectivo } \\
\text { con el PIB } \\
\text { inicial }\end{array}$ & $\begin{array}{c}\text { Valor } \\
\text { del flujo } \\
\text { de } \\
\text { efectivo } \\
\text { con el } \\
\text { PIB } \\
\text { final }\end{array}$ & $\begin{array}{l}\text { Variación } \\
\text { en el flujo } \\
\text { de efectivo }\end{array}$ \\
\hline-13.639 .479 & 586.801 & -13.719 .261 & 84.552 .075 & $-5 \%$ & $-4 \%$ & $\begin{array}{c}- \\
852.731 .51 \\
8,20\end{array}$ & $\begin{array}{c}- \\
608.911 . \\
224,58\end{array}$ & $28,59 \%$ \\
\hline-13.639 .479 & 586.801 & -13.719 .261 & 84.552 .075 & $-4 \%$ & $-3 \%$ & $\begin{array}{c}- \\
608.911 .22 \\
4,58\end{array}$ & $\begin{array}{c}- \\
406.612 . \\
681,11\end{array}$ & $33,22 \%$ \\
\hline-13.639 .479 & 586.801 & -13.719 .261 & 84.552 .075 & $-3 \%$ & $-2 \%$ & $\begin{array}{c}- \\
406.612 .68 \\
1,11\end{array}$ & $\begin{array}{c}- \\
242.315 \\
080,55\end{array}$ & $40,41 \%$ \\
\hline-13.639 .479 & 586.801 & -13.719 .261 & 84.552 .075 & $-2 \%$ & $-1 \%$ & $\begin{array}{c}- \\
242.315 .08 \\
0,55\end{array}$ & $\begin{array}{c}- \\
112.497 \\
615,67\end{array}$ & $53,57 \%$ \\
\hline-13.639 .479 & 586.801 & -13.719 .261 & 84.552 .075 & $-1 \%$ & $0 \%$ & $\begin{array}{c}- \\
112.497 .61 \\
5,67\end{array}$ & $\begin{array}{c}- \\
13.639 .4 \\
79,22\end{array}$ & $87,88 \%$ \\
\hline-13.639 .479 & 586.801 & -13.719 .261 & 84.552 .075 & $0 \%$ & $1 \%$ & $\begin{array}{c}- \\
13.639 .479 \\
22\end{array}$ & $\begin{array}{c}57.780 .1 \\
36,03\end{array}$ & $523,62 \%$ \\
\hline-13.639 .479 & 586.801 & -13.719 .261 & 84.552 .075 & $1 \%$ & $2 \%$ & $\begin{array}{c}57.780 .136 \\
03\end{array}$ & $\begin{array}{c}105.282 \\
037,32\end{array}$ & $82,21 \%$ \\
\hline-13.639 .479 & 586.801 & -13.719 .261 & 84.552 .075 & $2 \%$ & $3 \%$ & $\begin{array}{c}105.282 .03 \\
7,32\end{array}$ & $\begin{array}{c}132.387 \\
031,88\end{array}$ & $25,75 \%$ \\
\hline-13.639 .479 & 586.801 & -13.719 .261 & 84.552 .075 & $3 \%$ & $4 \%$ & $\begin{array}{c}132.387 .03 \\
1,88\end{array}$ & $\begin{array}{c}142.615 \\
926,96\end{array}$ & $7,73 \%$ \\
\hline-13.639 .479 & 586.801 & -13.719 .261 & 84.552 .075 & $4 \%$ & $5 \%$ & $\begin{array}{c}142.615 .92 \\
6,96\end{array}$ & $\begin{array}{c}139.489 \\
529,79\end{array}$ & $-2,19 \%$ \\
\hline-13.639 .479 & 586.801 & -13.719 .261 & 84.552 .075 & $5 \%$ & $6 \%$ & $\begin{array}{c}139.489 .52 \\
9,79\end{array}$ & $\begin{array}{c}126.528 . \\
647,62\end{array}$ & $-9,29 \%$ \\
\hline-13.639 .479 & 586.801 & -13.719 .261 & 84.552 .075 & $6 \%$ & $7 \%$ & $\begin{array}{c}126.528 .64 \\
7,62\end{array}$ & $\begin{array}{c}107.254 \\
087,67\end{array}$ & $-15,23 \%$ \\
\hline
\end{tabular}

Fuente: Elaboración propia. 
De acuerdo con los resultados presentados en la tabla anterior, se comprueba en el modelo que siempre que el crecimiento económico sea cero por ciento $(0 \%)$, el valor de los flujos de efectivo será igual al valor de la constante y por cada punto porcentual que varíe el PIB, los flujos de efectivo variarán en función a la ecuación de tercer orden. Las variaciones en el PIB se realizaron desde $-5 \%$ hasta $7 \%$, ya que en esta escala de variación PIB ha oscilado el rango de este indicador en los últimos 20 años para Colombia.

Según esto, si se desea establecer la variación que tendrán los flujos de efectivo del sector comercial en el departamento del Valle del Cauca, se debe utilizar esta variación obtenida, es decir, determinar los flujos de efectivo en función de la ecuación de tercer orden de acuerdo al PIB esperado.

Las variaciones en el PIB esperadas según los datos del Banco Mundial serán en 2014 del 4,6\% y en 2015 del 3,5\%. Por lo tanto, la variación del PIB será de $1,1 \%$ lo que significa que los flujos de efectivo deben variar en $-1,92 \%$. Y en 2016 el crecimiento en el PIB esperado es del 3,9\%, pues la variación con respecto al año 2015 es de $0,4 \%$, por lo cual los flujos de efectivo deben aumentar en 2,05\%. En 2017 el crecimiento en el PIB esperado es del 4,2\%, por tanto, la variación con respecto al año 2016 es de $0,3 \%$, por lo cual los flujos de efectivo deben aumentar en $0,49 \%$. Todo lo anterior se presenta en la siguiente tabla:

Tabla 10. Predicción de los flujos de efectivo para los años 2014 - 2017 para las empresas del sector comercial del departamento del Valle del Cauca.

\begin{tabular}{|c|c|c|c|}
\hline Período & $\begin{array}{c}\text { PIB } \\
\text { proyectado }\end{array}$ & $\begin{array}{c}\text { Valor esperado } \\
\text { de los flujos de } \\
\text { efectivo }\end{array}$ & $\begin{array}{c}\text { Variación de } \\
\text { los flujos de } \\
\text { efectivo }\end{array}$ \\
\hline 2014 & $4,6 \%$ & $142.117 .392,06$ & \\
\hline 2015 & $3,5 \%$ & $139.390 .941,40$ & $-1,918 \%$ \\
\hline 2016 & $3,9 \%$ & $142.252 .118,92$ & $2,053 \%$ \\
\hline 2017 & $4,2 \%$ & $142.946 .405,07$ & $0,488 \%$ \\
\hline
\end{tabular}

Fuente: Elaboración propia. 


\section{Conclusiones}

En la introducción del presente artículo, se enuncia que hay un vacío teórico en la relación que establecen las variables analizadas, según los autores que analizan la predicción de los flujos de efectivo mediante variables como son las ventas, los costos, algunos estados financieros, los flujos de efectivo históricos, entre otras variables. Sin embargo, se muestra en los resultados del presente trabajo que variables externas afectan también los resultados de los flujos de efectivo y que es posible establecer predicciones a través de estas variables, como es el crecimiento económico.

En la elaboración de la teoría que pueda respaldar la relación entre el crecimiento económico y los flujos de efectivo, se exploraron algunos autores que han hablado sobre el crecimiento económico, entre ellos Harrod (1939) que centró su tesis en que cuando un país presenta inestabilidades en su crecimiento se debe al propio intervencionismo estatal. Otro autor consultado fue Domar (1946) quien encontró que el crecimiento de la economía se centra en la demanda, de modo que, en la medida que esta se incrementa de igual forma lo hace la producción. Por su parte, Uzawa (1961) encuentra que el crecimiento económico se suscita por el libre movimiento de la mano de obra y del capital. Kaldor (1956) afirmó que el sector manufacturero es el motor del crecimiento de la economía. Pasinetti (1962) demostró que el crecimiento económico se da por el ahorro acumulado en las economías y la forma como en el largo plazo este ahorro se traslada para generar el crecimiento. Kalecki (1971) como Pasinetti y Kaldor, centró su teoría del crecimiento en el ahorro y la inversión por el estado y las empresas. Hicks (1969) quien demostró que el crecimiento de la economía se da cuando los factores de producción comienzan a escasear, los precios de estos aumentan, lo que estimula nuevas formas de producción.

Por su parte, Solow (1956), aunque en su teoría de crecimiento económico se acerca a postulados como los de Harrod (1939) y se asemeja a los fundamentos de autores como Pasinetti y Kalecki, presenta el crecimiento económico a partir de la acumulación de capital físico que está dado por una combinación entre capital humano y tecnología, de modo que el ahorro produce la liquidez necesaria que el sistema económico necesita para llevar a cabo las inversiones en capital que apuntarán al crecimiento de la economía a largo plazo. Por esto, la teoría base de la relación entre el crecimiento de la economía y los flujos de efectivo es la de este autor y que se expone en el gráfico número uno del presente trabajo.

Para establecer la relación entre los flujos de efectivo y el crecimiento económico, se buscaron autores que indicaran que la liquidez es la principal variable que conecta estos dos aspectos. Entre ellos, se tiene a Aceves y Martínez (2013) y Levine (1997), que señalan cómo un sistema financiero consolidado puede 
ofrecer la liquidez suficiente a los empresarios para que estos hagan uso de estos recursos en inversiones que impulsen el crecimiento económico.

Para presentar una mayor homogeneidad de los datos y demostrar que la liquidez es una herramienta clave de los empresarios para apuntalar sus inversiones, se presentan los flujos de efectivo operativos y se les suman a estos los flujos de efectivo de financiación, lo que mejora los resultados de las correlaciones y permiten un mejor pronóstico de los flujos de efectivo. Al agregarle a los flujos de efectivo la financiación, se demuestra que, al inyectarle liquidez a las empresas, estas disponen de mayores recursos para ser invertidos y se propicia el crecimiento de la economía, ya que los datos de correlación y predicción mejoraron frente a un escenario de menor liquidez Levine (1997).

Mediante el análisis cualitativo, se demuestra la relación que hay entre las variables crecimiento económico y flujos de efectivo, pues, en casos como el departamento del Valle del Cauca, durante 12 años, se observa que los hechos económicos de la región afectaron directamente los resultados de los flujos de efectivo para el sector comercial. Es decir, hay una asociación de hechos que explican las variaciones de los flujos de efectivo.

En el departamento del Valle del Cauca, el 53\% de los años presenta correlación directa entre las variables crecimiento económico y flujos de efectivo. Esta región se caracteriza porque el sector financiero, industrial, de servicios y comercial han sido los protagonistas para la generación del crecimiento de la economía regional (en ese orden). Sin embargo, en los últimos años sectores como la construcción han aumentado su participación en el PIB regional, pasando de un 3,11\% en el año 2000 hasta en un 7,37\% en el año 2014 apoyando al crecimiento del PIB, sin embargo, la participación del sector agropecuario ha perdido protagonismo al disminuir su cuota de aporte al PIB en un 1,1\% (del 6,4\% al 5,3\%).

En el desarrollo del presente trabajo, se realizó una comparación de correlación entre el crecimiento económico y los flujos de efectivo de operación del sector comercial. Además, se realizó la correlación entre el crecimiento económico y la sumatoria entre los flujos de efectivo de operación y financiación, llegando al $78 \%$, lo que significa que, al inyectarle liquidez a las empresas, estas tienen mayor disponibilidad de recursos que son destinados a la inversión que deriva en crecimiento económico, como lo indica Levine (1997). La correlación se logró gracias a la aplicación del modelo de regresión cúbica, que a su vez hace parte de un modelo polinómico, en este caso y es no lineal, es decir que las variables se ajustan mejor a una curva que a una recta (modelo lineal) y además se puede obtener la ecuación de tercer orden de dicha curva. El modelo presentado fue validado estadísticamente. 
Para el caso de la Economía, y en el momento de determinar los valores en los cuales el ingreso promedio es máximo o igual al ingreso marginal, la regresión polinómica es la que permite estimar de mejor manera el comportamiento de la función ingreso, para el caso del presente trabajo resulta ser válido el comportamiento en los estimados de los flujos de efectivo esperados (F.E.E.) mediante la ecuación de tercer orden, ya que presentarán una variación positiva o negativa en función de la variación del crecimiento económico determinándose de mejor manera el comportamiento de las variables.

A pesar de las amplias variaciones de flujo de efectivo interanuales mostradas por las empresas del sector y analizadas en el presente documento, el sector comercio para el departamento del Valle del Cauca, durante los últimos 15 años ha aportado al PIB total regional un promedio del $12,5 \%$, una cifra casi invariable de manera anual.

\section{Referencias}

Aceves, S., y Martínez, J. (2013). The financial system and his impact in the private sector dynamics. Revista Contaduría y administración, 58, 175 - 199.

Aranaz, M. (2002). SPSS para Windows. Madrid: Mc Graw Hill interamericana.

Atje, R., y Jovanovic, B. (1993). Stock market development and long - run growth. World Bank economic review, 10, 323 - 339.

Atwood, T., Drake, M., Myers, J. N., y L. A. M. (2011). Do earnings reported under IFRS tell us more about future earnings and cash flows? Journal of accounting and public policy, 30, $103-121$.

Banco Mundial. (2015). Real GDP growth at markets prices in percent and current account balance in percent of GDP, unless indicated otherwise. Recuperado el 12 de mayo de 2015, de Banco Mundial: www.worldbank.org/content/dam/worldbank/GEP/GEP2015a/pdfs/GEP2015a _chapter2_regionaloutlook_LAC.pdf

Bencivenga, V., Smith, B., y Starr, R. (1995). Transactions costs, technological choice, and endogenous growth. Journal of economic theory. Vol. 67, 153 - 177.

Catanach, A., Croll, D., y Grinaker, R. (2000). Taching intermediate financial accounting using a business activity model. American Accounting association, 15, 583 603.

Creswell, J. (2005). Educational research: planning, conducting and evaluating quantitative and qualitative research. Upper saddle river: Pearson education. 


\section{GABRIEL EDUARDO ESCOBAR ARIAS / JORGE IVÁN OSORIO MEJÍA}

DANE. (2014). Estadísticas, Producto Interno Bruto. Recuperado el 6 de mayo de 2015, de Departamento Administrativo Nacional de Estadística: www.dane.gov.co/index.php/esp/pib-cuentas-nacionales/investigacionesespeciales/77-cuentas-nacionales/cuentas-anuales/

Domar, E. (1946). Capital expansion, rate of growth, and employment. Econometrica, 14, $137-147$.

Escobar, G. (2014). Flujos de efectivo y entorno económico en las empresas de servicios de Risaralda, 2002 - 2011. Finanzas y politica económica, 6, 141 - 158.

Fisher, R. (1925). Statistical Methods for Research Workers. Oxford: Clarendon Press.

Francis, N., y Eason, P. (2012). Accruals and the naïve out - of- sample prediction of operating cash flow. Advances in accounting, 28, $226-234$.

García, O. (1999). Administración financiera fundamentos y aplicaciones. Cali: Editorial prensa Moderna.

Harrod, R. (1939). An essay in dynamic theory. The economic journal, 49, 14 - 33.

Hicks, J. (1969). A theory of economic history. Oxford: Claredon Press.

Kaldor, N. (1956). Alternative theories of distribution. The review of economic studies, $23,83-100$.

Kalecki, M. (1971). Selected essays on the dynamics of the capitalist economy. Cambridge: University Press Cambridge.

Lee, B. (1996). The contextual usefulness of financial statement analysis in predicting earnings growth and measuring market earnings expectations (PhD. Dissertation). Massachusetts: University of Massachusetts.

Lee, C. (2011). The effect of SFAS 142 on the ability of good will to predict future cash flows. Journal of accounting and public policy, 30, 235 - 255.

Legoria, J., y Sellers, K. (2005). The analysis of SFAS \# 109 usefulness in predicting future cash flows from a conceptual framework perspective. Research in accounting regulation, $18,143-161$.

León, O., y Montero, I. (2003). Métodos de Investigación en psicología y Educación. Madrid: McGraw-Hill.

Levine, R. (1997). Desarrollo Financiero y Crecimiento económico: Enfoques y temario. Journal of economic literature, 35, $688-726$. 
Lorek, K. S. (2014). Trend is statistically based quarterly cash - flow prediction models. Accounting forum, 38, $145-151$.

Luo, M. (2008). Unusual operating cash flows and stock returns. Journal of accounting and public policy, $26,420-429$.

North, D. (1990). Institutional change and economic performance. Cambridge MA: Cambridge University Press.

Ou, J., y Penman, S. (1989). Financial statement analysis and the prediction of stock returns. Journal of accounting and economics, 11, $295-329$.

Pasinetti, L. (1962). Rate of profit and income distribution in relation the rate of economic growth. The review of economic estudies, 29, $267-279$.

Robinson, J. (1952). The generalization of the general theory. En J. Robinson, The rate of interest and other essays (págs. 67 -142). London: McMillan.

Sampieri, R., Collado, C., y Lucio, P. (2006). Metodología de la Investigación. México DF: Mc Graw Hill.

Sancho, A., y Serrano, G. (2006). "Econometría de Económicas: apuntes para el tema 6”, Recuperado el 23 de noviembre de 2015. Obtenido de Universidad de Valencia: http://www.uv.es/ sancho/panel.pdf.

Shumpeter, J. A. (1912). The theory of economic development. Cambridge MA: Cambridge University Press.

Sloan, R. G. (1996). Do stock process fully reflect information in accruals and cash flows about future earnings? The accounting review, 71(3), 289-315.

Solow, R. (1956). A contribution to the theory of economic growth. Quarterly Journal of Economics, 78, 65-94.

Stober, T. (1992). Summary financial statement measures and analyst forecast of earnings. Journal accounting and economics, 15, 347 - 372.

Stober, T. (1993). The incremental information content of receivables in predicting sales, earnings and profit margins. Journal of accounting and finance, 8, $447-473$.

Supersociedades. (2015). SIREM, estados financieros sociedades año 1995 - 2014. Recuperado el noviembre de 2015, de Superintendencia de sociedades www.supersociedades.gov.co

Uzawa, H. (1961). On a two - sector economic growth model. The review of economic studies, $29,40-47$. 